\title{
Meteoric calcium
}

Calcium is found throughout the solar system, the Earth's crust and oceans, and is an essential constituent of cells, shells and bones - yet it is curiously scarce in the upper atmosphere. John Plane ponders on this 25-year-old mystery.

T he discovery in 1808 of element 20 is credited - just like its neighbours in the periodic table, potassium, sodium and magnesium, among others - to the English chemist Humphry Davy, who had developed an electrolysis technique for isolating elements from rocks. Calcium is essential for living organisms and takes part in various cellular processes. Yet, any present in the upper atmosphere is extra-terrestrial.

As interplanetary dust particles enter the atmosphere at very high speeds (11-72 $\left.\mathrm{km} \mathrm{s}^{-1}\right)$, collisions with air molecules induce flash heating, which often causes the particles to melt at around $1,800 \mathrm{~K}$, and the constituents to evaporate. This 'meteoric ablation' process typically occurs between 75 and $110 \mathrm{~km}$ of altitude, and directly injects metal atoms in the atmosphere.

Several of these meteoric metals can be observed from the ground using the 'lidar' technique: a pulsed laser is tuned to a spectroscopic transition of the metal of interest - blue light at $422 \mathrm{~nm}$ for calcium - and directed up into the atmosphere. This induces a resonance fluorescence from the metal, subsequently detected with a telescope. Lidar is an extraordinarily sensitive technique, able to detect calcium atom concentrations of less than 10 atoms $\mathrm{cm}^{-3}$ at a distance of $90 \mathrm{~km}$.

The first lidar measurements of calcium, made at the French Observatory of Haute Provence in $1985^{1}$, showed a peak concentration of around 20 atom $\mathrm{cm}^{-3}$ - about 200 times lower than the typical sodium atom concentration and 400 times lower than that of iron (see picture). This is in stark contrast with the Sun's photosphere, meteorites, or the Earth's crust, where calcium is nearly as abundant as sodium, and only 16 times more scarce than iron. Where does the enormous relative depletion of calcium in the upper atmosphere come from?

There are only three possibilities: calcium is already depleted in the interplanetary

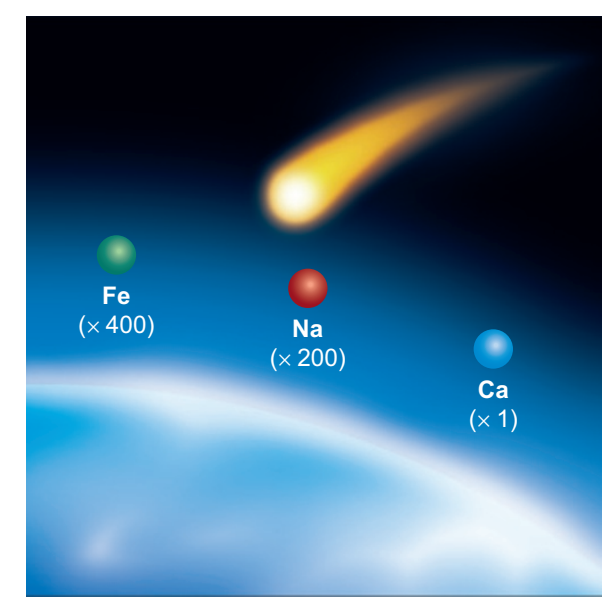

A layer of metallic atoms, produced between 80 and $100 \mathrm{~km}$ in the Earth's atmosphere by the ablation of cometary dust particles. The concentration ratio of $\mathrm{Ca}: \mathrm{Na}: \mathrm{Fe}$ is about 1:200:400.

dust particles entering the atmosphere; it does not ablate efficiently from meteors; or its atmospheric chemistry is quite different from that of sodium and iron. Until recently, our knowledge of the composition of interplanetary dust particles was restricted to meteorites recovered on the surface of the Earth. No significant calcium depletion was observed - but these samples survived atmospheric entry (the definition of meteorites), and so the bulk of the incoming dust might already have been calcium-poor. The current consensus is that most interplanetary dust particles that reach the Earth originate from comets. In 2006, the NASA Stardust spacecraft collected particles from Comet 81P/Wild 2 - unfortunately, their analysis revealed no depletion of calcium ${ }^{2}$.

The second possibility is that the ablation process favours relatively volatile elements (sodium and iron) compared with refractory ones (calcium). This is consistent with thermodynamics, because calcium forms very stable oxides in silica melts, and in fact there is evidence that such a 'differential ablation' does occur. However, models predict a relative depletion of calcium with respect to sodium of only about a factor of 3 , rather than the observed factor of 200 (ref. 3).

Is the atmospheric chemistry of calcium different from sodium and iron? As a result of laboratory studies, we now have a fairly clear idea of why such reactive metallic elements occur in their reduced form in what is a highly oxidizing atmosphere ${ }^{4}$. The key factor is that around $85 \mathrm{~km}$ of altitude, the photolysis of $\mathrm{O}_{2}$ and $\mathrm{H}_{2} \mathrm{O}$ by extreme ultraviolet radiation leads to high concentrations of oxygen and hydrogen atoms. Hydrogen and oxygen atoms attack metallic compounds such as hydroxides and oxides, reducing them back to metal atoms. However, the relevant reaction rates of calcium and its compounds are quite similar to those of the other metals ${ }^{5}$, which means that the difference in reactivity accounts for no more than a factor of 5 in the relative abundance of calcium to sodium.

Thus, there remains an unexplained depletion of calcium by at least one order of magnitude. This is probably due to our poor understanding of the extreme chemistry that occurs during meteoric ablation, combined with continuing (and controversial) uncertainties about the velocity and mass distributions of interplanetary dust particles, which depend on their cometary and asteroidal origins. The calcium mystery may therefore end up telling us a great deal more about the evolution of the solar system.

JOHN M. C. PLANE is Professor of Atmospheric Chemistry at the University of Leeds, Leeds LS2 9JT, UK.

e-mail: j.m.c.plane@leeds.ac.uk

\footnotetext{
References

1. Granier, C., Jegou, J. P., \& Megie, G. Geophys. Res. Lett. 12, 655-658 (1985)

2. Flynn, G. J. et al. Science 314, 1731-1735 (2006)

3. Vondrak, T., Plane, J. M. C., Broadley, S. \& Janches, D. Atmos. Chem. Phys. 8, 7015-7031 (2008).

4. Plane, J. M. C. Chem. Rev. 103, 4963-4984 (2003).

5. Broadley, S. L. \& Plane, J. M. C. Phys. Chem. Chem. Phys. 12, 9095-9107 (2010).
} 